\title{
Lung sonography as a predictive tool for extubation failure in intensive care.
}

\author{
khoudi R., Charrada H., Feki M., Lamouchi A., Mili S., Benali M. \\ Mohamed Tahar Mâamouri Hospital, Dept of Anaesthesiology \& Intensive Care, Nabeul, Tunisia.
}

\section{Background and Goal of Study:}

Weaning from mechanical ventilation (MV) in intensive care is a major issue. The decision of extubation based on clinical and biological criteria has a rate of failure between $10-20 \%$. It is then important to search for new criteria to predict better the failure or the success of a respiratory withdrawal attempt. The aim of this study was to assess the relevance of the Lung Ultrasound score (LUS) to predict the failure or success of extubation.

\section{Patients and methods:}

It is a prospective study including intubated patients ventilated for more than 48 hours and that met the international consensus criteria. The age less than 18 years, patients with tracheostomy, paraplegic patient with medullar level above T8 and patients with planned prophylactic noninvasive ventilation were the exclusion criteria. The spontaneous breathing trial (SBT) was performed through a T-Tube. Patients who successfully passed the SBT were extubated and followed up for 48 hours. Then we classified patients into two groups: the post-extubation success group (Group1) or post-extubation distress group (Group2). Lung ultrasound echocardiography LVEF (reports E/Ea and E/A) and clinical biological parameters were performed during spontaneous pressure support ventilation (PSV) and at the end of a $60 \mathrm{~min}$ SBT. We examined six regions of the left and the right of the chest. Four ultrasound aeration patterns were defined: Normal aeration and presence of A lines [class1], a moderate loss of aeration B1 lines [class2], a severe loss of aeration B2 lines [Class3] and lung consolidation [Class4].

The primary outcome parameter was LUS after SBT. Secondary outcome parameters were the E/Ea, the E/A, the LUS after PSV and mortality. All Statistical analysis were performed using IBM SPSS 20. The Receiving Operating Curve was used to determine the Cut-OFF value of LUS.

\section{Results:}

Thirty-Five patients were included. Three patients were excluded from this study. Thirty-two patients have successfully passed the SBT and then were extubated. Twenty-four (75\%) were finally weaned at 48 hours. Eight (25\%) patients were re-intubated or have benefited from a NIV before 48 hours. The LUS after SBT was significantly higher in Group2 (19.7 \pm 2.5$)$ vs Group1 (11.8 \pm 2.6$)$ with $\mathrm{p}<10^{-3}$. The LUS after PSV was significantly higher in Group2 $(16.25 \pm 2.1)$ vs Group1 $(9.3 \pm 3)$ with $\mathrm{p}=0.01$. A score of LUS in SBT more than 16 predicted weaning failure with a sensitivity of $87.5 \%$ and a specificity of $83.3 \%$ $(\mathrm{AUC}=0.99)$. A LUS lower than 14 predict success with a high sensitivity and specificity in COPD subgroup. The E/A was significantly higher in Group2 $(2 \pm 0.7)$ vs Group1 (1.08 \pm 0.2 ) with $\mathrm{p}=0.021$ and $\mathrm{E} / \mathrm{Ea}$ was also higher in Group2 $(10,6 \pm 2.6)$ vs Group1 $(6.9 \pm 0.5)$ with $p=0.006$.

A statistically significant difference between groups regarding age Group2 (57 \pm 16 years) vs Group1 (31 \pm 12 years) with $\mathrm{p}=0.002$, history of COPD Group2 85.7\% vs. Group1 $14.3 \%$ with $\mathrm{p}=0.001$.
There are no difference between two groups in terms of sex, Sequential Organ Failure Assessment score, metabolic parameters (serum calcium level, serum phosphate and magnesium, protidaemia), complications (ARDS, ventilatorassociated pneumonia, acute renal failure) and length of stay in the ICU. Mortality was significantly higher in Group2 (75\%) vs group $1(4.2 \%)$ with $\mathrm{p}<10^{-3}$. The overall mortality was $21.8 \%$.

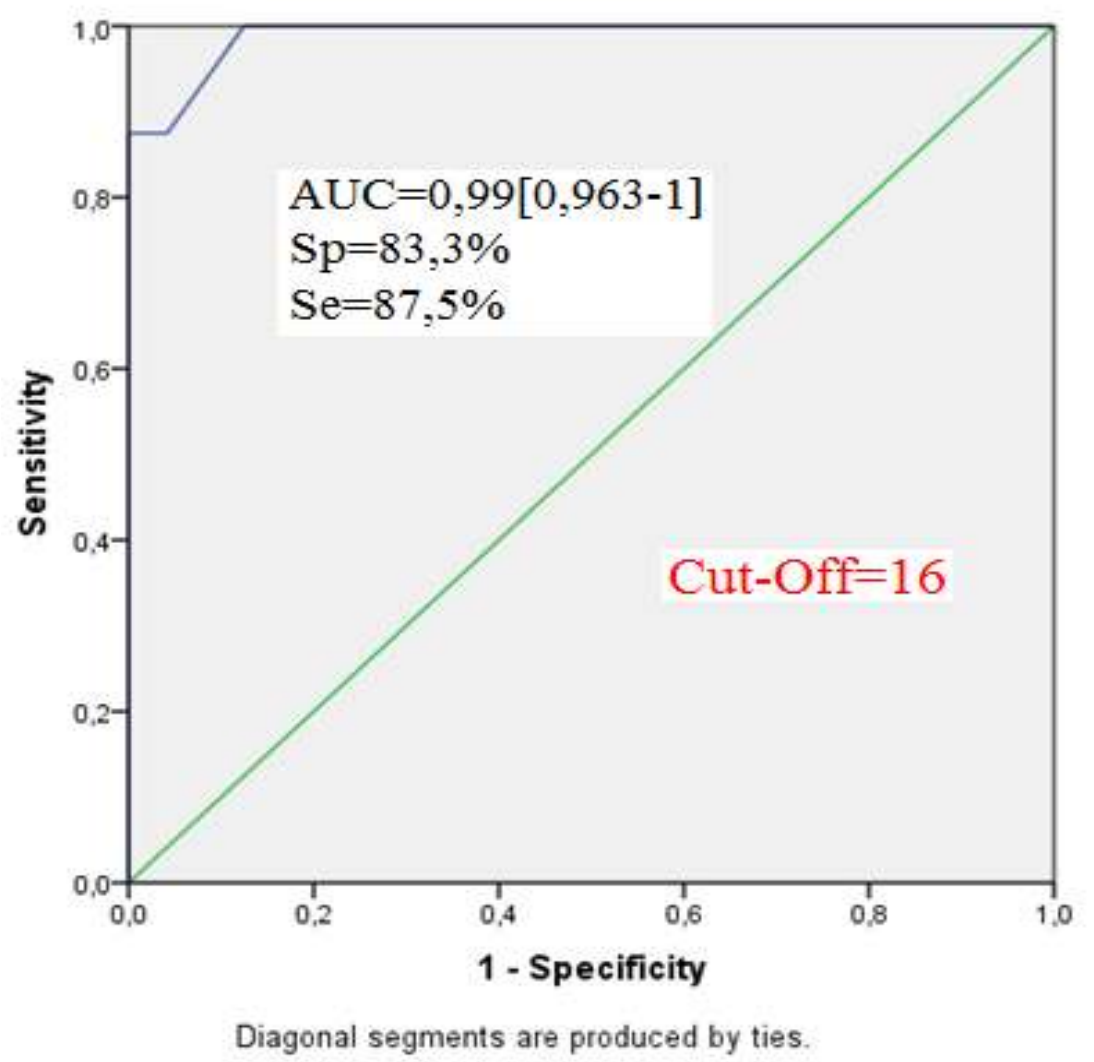

\section{Discussion:}

In this prospective observational study, weaning from MV was associated with significant lung derecruitment as assessed by transthoracic lung ultrasound. In patients who successfully passed the SBT, a lung ultrasound score $\geq 16$ at the end of SBT is highly predictive of post-extubation distress. As in our study, Soummer et al provided that an end SBT LUS $<13$ was predictive of postextubation success and an end-SBT LUS $>17$ was predictive of post-extubation distress [1]. The second major finding of this study shows that the diastolic dysfunction assessed by measurement of $\mathrm{E} / \mathrm{A}$ and $\mathrm{E} / \mathrm{Ea}$ was associated with weaning failure. As in our study, Lamia et al and Caille et al, found that elevated reports E/A and E/Ea could predicted an elevation in PAOP at the end of SBT and could then identify patients at risk of weaning failure[2 ,3]. The weaning process represents an additional work for the cardiovascular system and increases the venous return and afterload.

\section{Conclusion:}

The determination of the variation of pulmonary ventilation assessed by the lung ultrasound score predict well the failure or the success of respiratory withdrawal attempt until the $48^{\text {th }}$ hours especially among high-risk patients.

\section{References:}

[1]Crit Care Med. $2012 ; 40(7): 2064-72$.

[2]Crit Care Med. $2009 ; 37(5): 1696-701$.

[3]Crit Care. $2010 ; 14(3): \mathrm{R} 120$

Copyright @ 2016 Doctor Charrada Hedi (hedi1med@yahoo.fr; Phone +216 22908288 ) 